\title{
Impact of Pill Sharing on Drug Resistance Due to a Wide-Scale Oral Prep Intervention in Generalized Epidemics
}

Dobromir Dimitrov ${ }^{1 *}$, Marie-Claude Boily ${ }^{2}$, Benoît R. Mâsse ${ }^{3}$ and Elizabeth R. Brown ${ }^{1}$

${ }^{1}$ Vaccine \& Infectious Disease Division, Fred Hutchinson Cancer Research Center, Seattle, USA

${ }^{2}$ Department of Infectious Disease Epidemiology, Faculty of Medicine, Imperial College London, London, UK

${ }^{3} \mathrm{CHU}$ Sainte-Justine Research Centre, University of Montreal, Montreal, Quebec, Canada

\begin{abstract}
Background: The first antiretroviral drug (Truvada) to be used as a pre-exposure prophylaxis (PrEP) in preventing HIV transmission is about to be approved. Behavioral studies suggest that a portion of users may share anti-retroviral drugs with sex partners, family, or friends. Pill sharing will decrease PrEP efficacy and adherence level, and potentially create an environment favorable for the development of drug resistance. We aim to evaluate the potential impact of pill sharing on the PrEP effectiveness and on the rates of drug-resistance development in heterosexual populations.

Methods: A transmission dynamic model was used to assess the population-level impact of oral PrEP. The fractions of new HIV infections prevented (CPF), drug resistance prevalence and the proportion of new infections in which drug-resistant HIV is transmitted (TDR) are evaluated over fixed time periods. The influence of different factors on CPF and TDR is studied through simulations, using epidemic parameters representative of the countries in Sub-Saharan Africa.

Results: Without pill sharing, a $70 \%$ efficacious PrEP used consistently by $60 \%$ of uninfected individuals prevents $52.8 \%$ (95\% Cl 49.4\%-56.4\%) of all new HIV infections over ten years with drug-resistant HIV transmitted in $2.2 \%$ of the new infections. Absolute CPF may vary by $9 \%$ if up to $20 \%$ of the users share PrEP while the level of TDR and total resistance prevalence may increase by up to 6 -fold due to pill sharing in some intervention scenarios.

Conclusion: Pill sharing may increase the PrEP coverage level achieved in the population but it also affects the PrEP efficacy for the users who do not follow the prescribed schedule. More importantly, it creates a pool of untracked users who remain unreached by the effort to avoid sub-optimal PrEP usage by infected people. This increases substantially the potential risk of drug resistance in the population.
\end{abstract}

Keywords: HIV; Pill sharing; Pre-exposure prophylaxis

\section{Introduction}

The HIV pandemic causes more than 1.8 million deaths each year [1]. Although declining in many countries, HIV incidence remains high with an estimated total of 2.6 million new infections in 2009, most of which occur in Sub-Saharan Africa [1]. In addition, the number of people living with HIV/AIDS in the world is rising steadily, due in part to increased survival following expanded access to antiretroviral treatment (ART). The prevalence of HIV varies greatly worldwide but is highest in Southern Africa with prevalence rates in the heterosexual population exceeding 10\% [1]. There is also evidence of substantial HIV rates among men-having-sex-with-men (MSM) in low and middle income countries, including Africa [1-3]. Without significant reduction in the number of new HIV infections, the burden on the most affected countries in Sub-Saharan Africa will continue to grow to unsustainable levels. In order to substantially reduce or locally eliminate the magnitude of the epidemic, a more aggressive global effort to develop, test, and implement interventions to reduce HIV transmission is needed. In the past two years four proof-of-concept trials demonstrated that daily or coitally dependent use of pre-exposure prophylaxis (PrEP) in the form of pill (oral PrEP) or topical gel (vaginal microbicides) is $40 \%-70 \%$ effective in preventing HIV acquisition [4-7]. Although other recently concluded or ongoing trials did not confirm these findings $[8,9]$, the advisory panel to the Food and Drug Administration (FDA) in the United States already recommended one antiretroviral drug (Truvada) to be used as PrEP in US [10] and guidelines for the safe use of preexposure prophylaxis among MSM have recently been published by the Center for Diseases Control (CDC) and Southern African HIV Clinicians Society [11]. When approved PrEP products will extend the pool of options for HIV prevention available to state and local health officials for designing wide-scale implementation programs. One of the greatest threats that could endanger the successful addition of PrEP to HIV prevention program is that PrEP users who become HIV-infected may acquire drug resistance (ADR) and increase the risk of transmitted drug resistance (TDR). Currently, few resistance cases were reported in the completed clinical trials but PrEP users becoming HIV-infected were taken off PrEP very quickly. Studies in which short course of tenofovir have been offered to HIV-positive individuals also did not observe development of resistance $[12,13]$. This may lead to the optimistic conclusion that the resistance due to PrEP use will be substantially smaller problem compared to the resistance from ART. However, the implementation of PrEP outside clinical trial settings is unlikely to require monthly monitoring of the HIV status of PrEP users. Some efforts to restrict the usage of PrEP by HIV-infected individuals will likely be incorporated in a wide-scale PrEP intervention program through initial and periodic HIV screening of the prescribed users but

${ }^{*}$ Corresponding author: Dobromir Dimitrov, Ph.D., Vaccine \& Infectious Disease Division, Fred Hutchinson Cancer Research Center, Seattle, WA, USA, Tel: 206.667.1933; Fax: 206.667.4812; Email: dobromir@scharp.org

Received May 26, 2012; Accepted July 05, 2012; Published July 08, 2012

Citation: Dimitrov D, Boily MC, Mâsse BR, Brown ER (2012) Impact of Pill Sharing on Drug Resistance Due to a Wide-Scale Oral Prep Intervention in Generalized Epidemics. J AIDS Clinic Res S5:004. doi:10.4172/2155-6113.S5-004

Copyright: $\odot 2012$ Dimitrov D, et al. This is an open-access article distributed under the terms of the Creative Commons Attribution License, which permits unrestricted use, distribution, and reproduction in any medium, provided the original author and source are credit ed. 
with less intensity than what can be achieved in a clinical trial. Even if successful, these measures will not prevent individuals from sharing their prescription with others (pill sharing) or obtaining PrEP pills from the black market.

Experts warn that limited access to antiretroviral drugs for HIV treatment in resource-constrained countries may pressure individuals to share their antiretroviral drugs with others leading to sub-optimal treatment and higher risk of therapeutic failure [14]. For example, a clinical trial among pregnant women in Kenya evaluating the compliance to Thai-CDC regimen (zidovudine twice daily from 36 weeks gestation and 3-hourly during labor) reported that some women used more than the prescribed number of pills and admit sharing with family members [15]. In a home-based AIDS care program in Uganda, $6 \%$ of the participants report sharing their antiretroviral drugs with others [16]. A behavioral intervention trial in US (Project MIX) estimates that $2-4 \%$ of the HIV negative MSM informally used ART prescribed to their infected partners to reduce their risk of HIV infection [17]. Surveys of willingness to use PrEP products in seven countries demonstrated that the majority of participants have intention to share PrEP if it is prescribed to them [18]. The effects of pill sharing on the expected public health impact of PrEP interventions have not been studied to date. None of the mathematical models used to simulate interventions of oral or topical PrEP have incorporated pill sharing [1929]. A recent modeling study concludes that increased risk behavior and rate of emergence of drug-resistance while on PrEP have almost no effect on the number of new infections due to exposure to resistant strains [20] because of the short time window for individuals, who acquire resistance while on PrEP, to transmit resistant HIV. This result is based on the assumption in the study that all users are tested for HIV at least twice per year and immediately stop using PrEP as soon as they are found positive, which may be difficult to achieve in real life settings.

In this paper we evaluate the potential impact of pill sharing on the PrEP effectiveness in preventing HIV transmission and to predict the rates of drug-resistance due to PrEP use in heterosexual populations. We develop and analyze a transmission dynamic model of HIV epidemic to assess the population-level impact of oral PrEP in a variety of intervention scenarios in generalised epidemics settings characterised by high HIV prevalence. We compare the cumulative fraction of new HIV infections prevented, the cumulative fraction of transmitted drug resistance and the prevalence of drug resistance for scenarios with and without pill sharing over periods of PrEP use up to 20 years. The effects of intervention parameters on the PrEP impact are studied with a multivariate sensitivity analysis.

\section{Methods}

\section{Transmission model}

We present a compartmental mathematical model of HIV transmission in heterosexual population to study the impact of pill sharing on oral PrEP interventions (Figure 1). The population is stratified in compartments by gender (men, women) and by HIV status as susceptible, infected with wild-type HIV, infected with drug-resistant HIV through transmission, individuals who developed (acquired) resistance on PrEP and individuals who develop AIDS. Men and women who become sexually active join the community at constant rates, which are selected to balance the departure rate in a non-infected population. The rates at which men and women acquire HIV-infection, i.e., forces of infections for different classes are derived from standard binomial models based on the number of partners per susceptible person, the number of sex acts per partnership, the fraction of sex acts protected by condoms (assumed independent of PrEP use), the protection or effectiveness provided by PrEP (if used) and the HIV acquisition risk per vaginal act for men and women. We do not explicitly model anal intercourse because oral PrEP (unlike vaginal microbicide, [30]), can be assumed to be efficacious during all modes of exposure, which means that our impact estimate is not diluted by the practice of anal sex.

\section{Epidemic settings}

We use sexual behavior and epidemiological data representative for the Southern Africa $[1,31,32]$ to identify realistic ranges for the preintervention parameters of our model. Acquisition probabilities per vaginal act with HIV infected partner for men and women are obtained from a meta-analysis of the observational data from developing countries [33]. Demographic and sexual behavior characteristics including average number of partners per year, frequency of sex acts, fraction of protected sex acts, and time to remain sexually active are estimated from WHO data for South Africa and other published analyses $[31,32,34]$. Epidemic parameters are initially sampled uniformly from these ranges (Table 1, part A) and 1000 parameter sets are selected to produce persistent epidemics (i.e., basic reproductive number $\mathrm{R}_{0}$ greater than one) and HIV prevalence between $1 \%$ and $35 \%$ in absence of PrEP intervention. The histogram of the parameter sets with respect to the HIV prevalence (Figure $2 \mathrm{~A}$ ) shows that the vast majority of the simulated epidemics represent high prevalence settings with $84 \%$ of the simulations yielding prevalence of $10 \%$ or higher.

\section{PrEP efficacy and development of resistance}

The results from concluded clinical trials suggest that when used consistently oral PrEP provides protection against HIV acquisition and therefore reduces the susceptibility of the users [5-7]. Here, PrEP efficacy per act is explored in the range of $50 \%-90 \%$ which is a conservative estimate of the protection provided to perfect adherers. Age structure

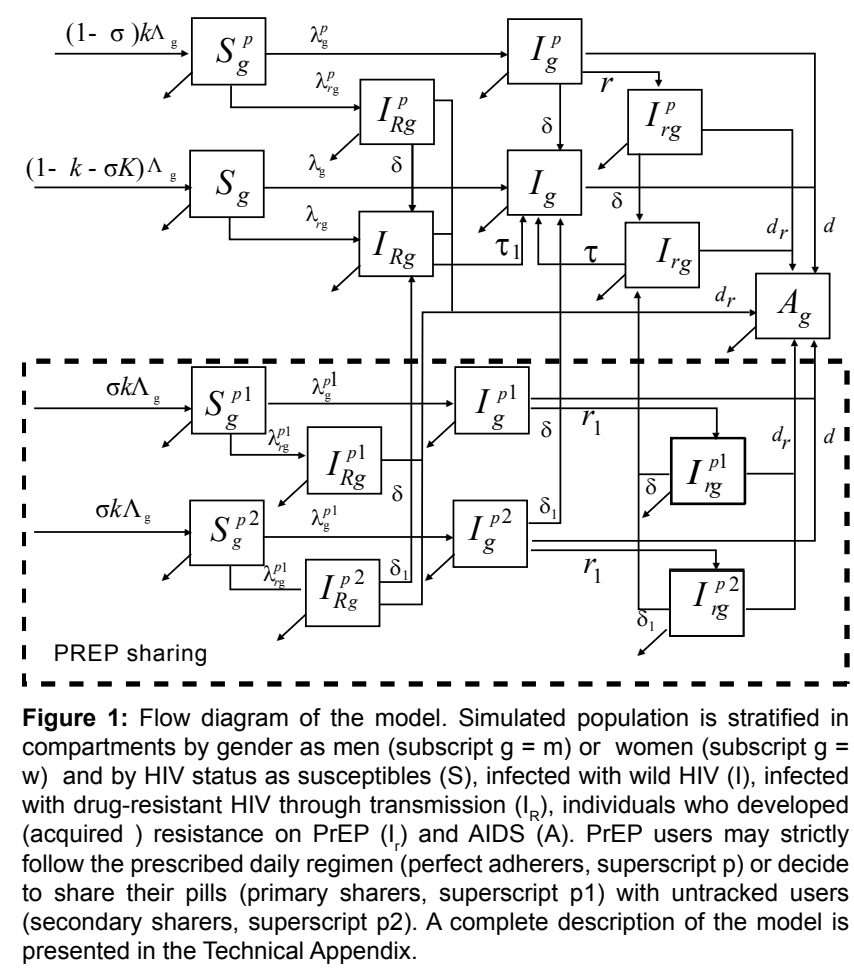



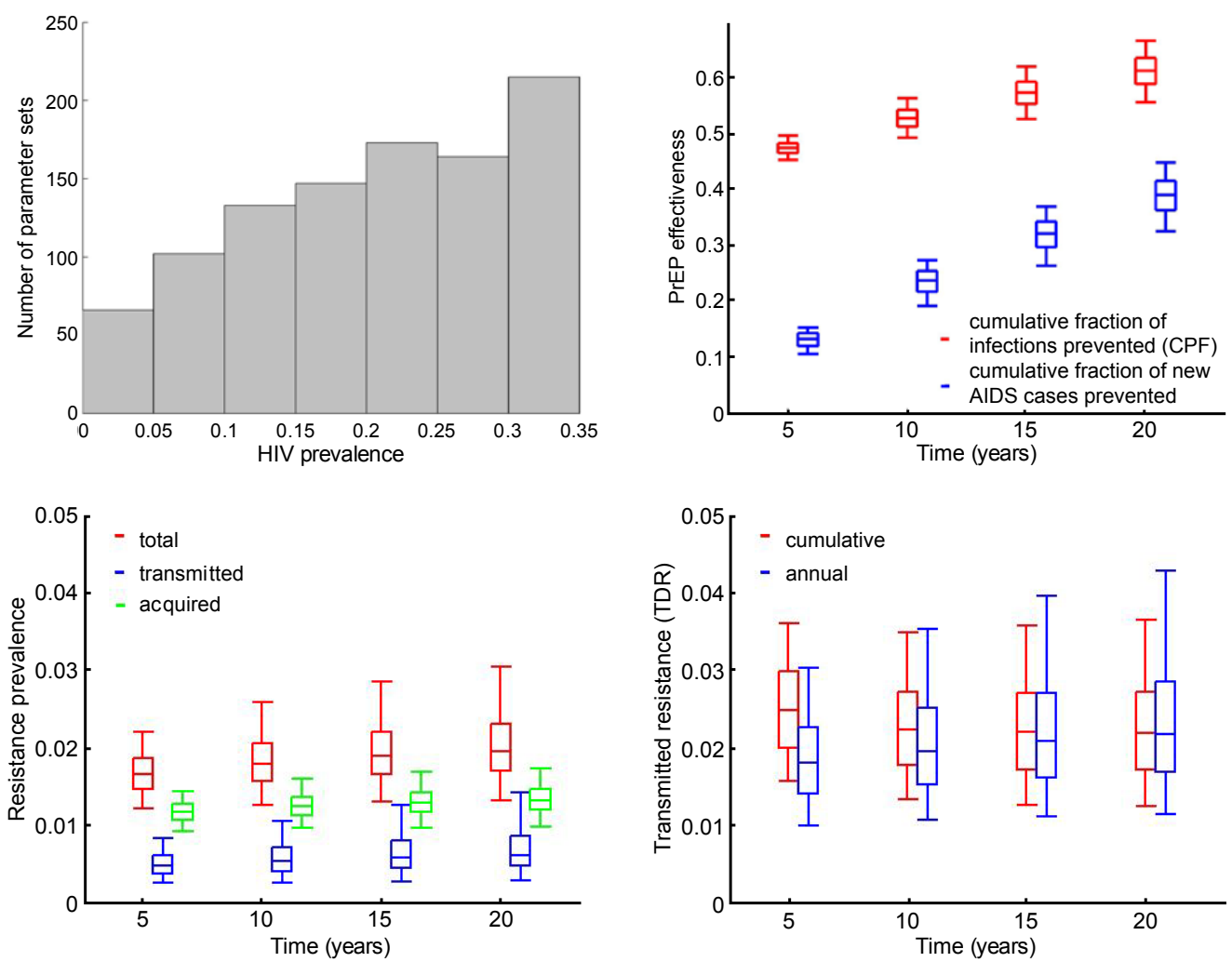

Figure 2 Results for the baseline scenario without pill sharing. A) Histogram of the HIV prevalence of 1,000 simulated epidemics without PrEP. B) Total (men and females combined) cumulative fraction of cumulative fraction of new infections and AIDS cases prevented by PrEP over up to 20 years after PrEP introduction. C) Resistance prevalence over up to 20 years after PrEP introduction D) Total fraction (cumulative and annual) of transmitted resistance over up to 20 years after PrEP introduction the box plots (median, 2.5th, 25th, 75th, 97.5 th percentiles) reflect the variation in impact estimates based on 1,000 epidemic parameters sets used. Intervention parameters are fixed on their baseline values from Table 1, part B.

of the population was not considered in the model since no significant association between efficacy of PrEP and age has been reported to date [5]. Theoretically, ARV-based PrEP may suppress the viral load increase of HIV-positive users and therefore reduce their risk of transmitting the virus. Experimental studies with macaques showed that the animals which become infected when on PrEP had significantly reduced viral load compared to controls [35]. Studies of tenofovir use have by HIVpositive individuals also associated it with low-level viremia $[12,13,36]$. This supports a potential role of PrEP in reducing infectiousness which we incorporated in our protection mechanism. Since clinical trials have strongly linked the level of adherence with the level of effectiveness [37-39], we assume that the protection conferred by PrEP is reduced if they skip doses (suboptimal regimen) or if they split pills (suboptimal dosage). Consistent PrEP use which is not able to suppress completely HIV viremia after HIV acquisition will lead to development of drug resistance [40,41] and that may reduce substantially the expected benefits from PrEP use. We assume 50\% to 100\% loss of protection when exposed to drug-resistant HIV compared to wild-type. If PrEP use is interrupted after development of resistance, i.e. the selective pressure is removed, the wild-type HIV is expected to regain dominance after a period which is much longer for individuals who acquire drug-resistant HIV through transmission (TDR) compared to those who develop resistance when on PrEP (ADR). Transmissibility of the resistant HIV is reduced by up to $50 \%$ due to fitness cost $[42,43]$.

\section{Pill sharing}

In our model, PrEP users either strictly follow the prescribed daily regimen (perfect adherers) or share their pills (primary sharers) with untracked users (secondary sharers). A primary sharer is a user who has a prescription for PrEP and shares a fraction of his/her pills with a family member, partner, friend (secondary user), on a regular basis. For simplicity, we assume that primary and secondary sharers follow the same regimens of PrEP use and are exposed to the same drug dosage which accommodates scenarios of reduced adherence in which sharers alternate to take pills and scenarios of reduced dosage in which sharers physically split each pill in half but follow the prescribed schedule. As a result of this sharing mechanism PrEP adherence and efficacy are equally affected for both the primary and secondary sharers. Prescribed users (perfect adherers and primary sharers) undergo initial and consequent periodic HIV testing and if found HIV-positive immediately stop PrEP use. Secondary sharers are assumed to be tested less frequently and to remain on PrEP significantly longer after becoming infected.

\section{Intervention scenarios}

For each parameter set, we simulate epidemics without PrEP and with intervention assuming no pill sharing. In this baseline scenario PrEP effectiveness is fixed at $70 \%$ to match the estimates obtained from Partners Study and approximations for full adherers from iPrEx trial. We simulate $60 \%$ instantaneous uptake of PrEP in the uninfected population while the initial usage by HIV infected individuals is $12 \%$ 


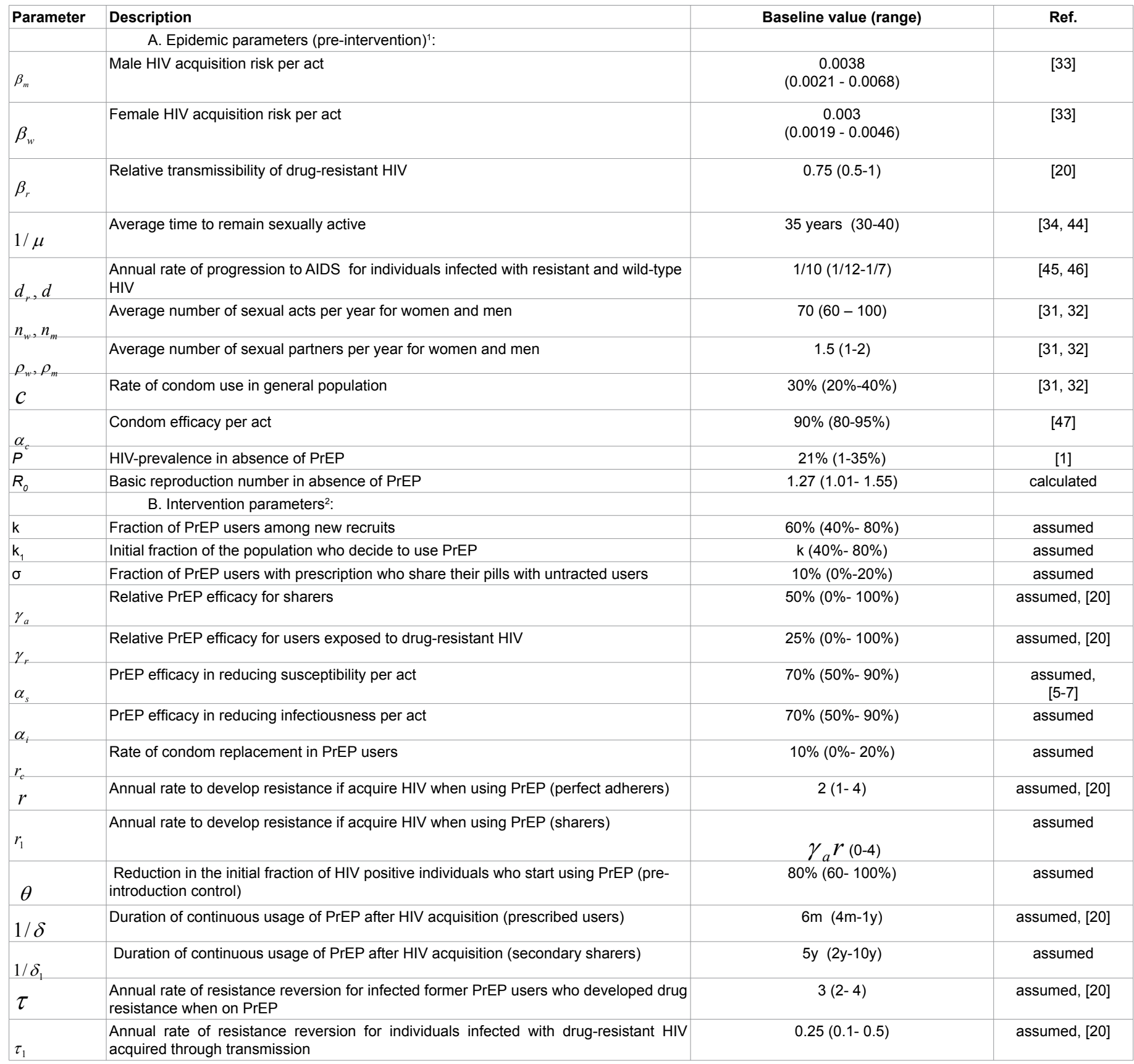

${ }^{1}$ Ranges for epidemic parameters are sampled uniformly to obtain parameter sets which are filtered to select 1000 epidemics with R0>1 and HIV prevalence between $1 \%$ and $35 \%$ in absence of PrEP.

${ }^{2}$ Ranges for intervention parameters are used in multivariate sensitivity analysis.

Table1: Parameter values and ranges.

(relative reduction of $80 \%$ compared to HIV negative). Prescribed users in the baseline scenario are tested for HIV annually and therefore remain on PrEP for 6 months after HIV acquisition on average. Individuals using PrEP after HIV infection are assumed to develop resistance over an average period of 1 year while the effectiveness of PrEP against drug-resistant HIV is fixed on $25 \%$ as in [20]. The influence of our choice of intervention settings on the evaluation outcomes is studied in multivariate sensitivity analysis using ranges described in Table 1, part B.

25 pill-sharing scenarios in which the fraction of prescribed PrEP users who share their pills is varied between $0 \%$ and $20 \%$ while the relative PrEP efficacy for sharers compared to perfect adherers is varied between $0 \%$ and $100 \%$ ( $0 \%$ means no protection while $100 \%$ implies the same level of protection as for perfect adherers). The rest of the intervention parameters are fixed on their baseline values described above (Table 1, part B).

\section{PrEP evaluation metrics}

The effectiveness and drug resistance risks associated with each intervention are evaluated through several different metrics 

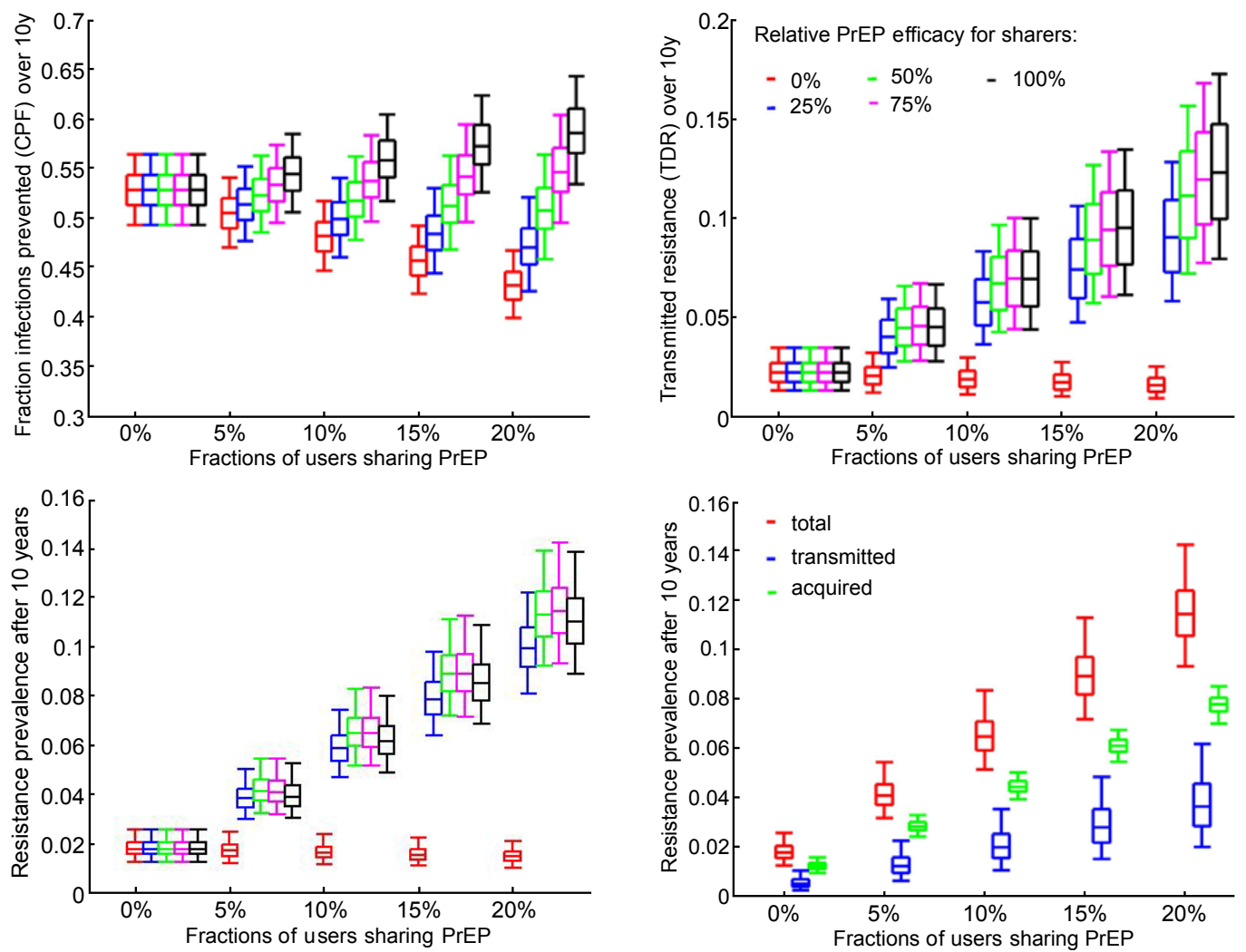

Figure 3: Results for scenarios with up to $20 \%$ of users who share PrEP and different levels of relative PrEP efficacy for sharers compared to perfect adherers. A) Total (men and females combined) cumulative fraction of of new infections prevented by PrEP over 10 years of intervention; B) Cumulative fraction of transmitted resistance over 10 years of intervention; C) Total resistance prevalence (acquired and transmitted combined) after 10 years of intervention and D) resistance prevalence by mode after 10 years of intervention assuming $75 \%$ relative protection for sharers the box plots (median, 2.5 th, 25 th, 75 th, 97.5 th percentiles) Reflect the variation in impact estimates based on 1,000 epidemic parameters sets used. Intervention parameters are fixed on their baseline values from Table 1, part B.

(quantitative indicators). The benefits from PrEP use are evaluated by the fraction of the cumulative infections $(\mathrm{CPF})$ and the fraction of new AIDS cases prevented over different periods after PrEP introduction. $\mathrm{CPF}$ is calculated as the difference of the cumulative number of new HIV infections in absence and presence of PrEP divided by the number of new infections in absence of PrEP.

The resistance risk due to PrEP is studied through the cumulative fraction of new infections in which drug-resistant HIV is transmitted (TDR), calculated on the basis of total number of new HIV infections, and the expected prevalence of drug resistance after fixed periods of PrEP usage.

\section{Results}

The results from our baseline scenario (no pill sharing) over time are presented in Figure 2. A 70\% effective oral PrEP intervention used by $60 \%$ of the population (male and females) is predicted to prevent about 52.8\% (95\% CI 49.4\%-56.4\%) of all new HIV infections over 10 years following its introduction (10-year $\mathrm{CPF}$ ) if perfect adherence is assumed (Figure 2B) . CPF increases to 61.3\% (95\% CI 55.7\%-66.7\%) if evaluated over 20 -year period. The use of PrEP leads to $23.8 \%$ (95\% CI $19.2 \%-27.4 \%$ ) and $39.2 \%$ (95\%CI 32.6\%-44.9\%) of the new AIDS cases prevented over 10-year and 20-year period, respectively. With no pill sharing and periodic testing of prescribed users every year the expected drug resistance due to PrEP remains under control with total resistance prevalence (Figure 2C) and fraction of infections in which resistant
HIV is transmitted (Figure 2D) estimated between 1.5\%-2.5\% over 20 years of PrEP use.

The effects of pill sharing on the evaluation metrics over 10 years of PrEP use is presented in Figure 3. The estimate of the 10-year CPF increases to $55.8 \%$ (95\% CI 51.7\%-60.4\%) and 58.6\% (95\% CI 53.5\%$64.3 \%$ ) in populations with $10 \%$ and $20 \%$ pill sharing respectively (Figure 3A, black), assuming no efficacy reduction for sharers (i.e. same efficacy as for perfect adherers). This positive impact of pill sharing is due to the increased coverage combined with the unaltered protection for the sharers. However, the CPF decreases if PrEP efficacy for sharers is reduced. It drops below the CPF obtained in absence of sharing if efficacy is reduced by more than $50 \%$. For example, If PrEP efficacy for sharers is $25 \%$ of that for full adherers then the absolute 10 -year CPF is reduced by $3 \%$ and $6 \%$ in the scenarios with $10 \%$ and $20 \%$ sharing (Figure 3A, blue), compared to the baseline scenario assuming no sharing. In the worst case scenario in which $20 \%$ of the prescribed PrEP users share pills and PrEP is completely ineffective for those sharers, the intervention loses about $20 \%$ of its effectiveness over 10 years due to pill sharing.

The fraction of new infections in which drug-resistant HIV is transmitted over 10 years (10-year TDR) is also affected if pill sharing practices are assumed (Figure $2 \mathrm{~B}$ ). The expected level of 10-year TDR increases by up to 6 -fold in scenarios where the PrER efficacy for sharers is between $25 \%$ and $100 \%$ of the efficacy for perfect adherers (Figure 3B). The expected TDR declines very marginally only if PrEP is 

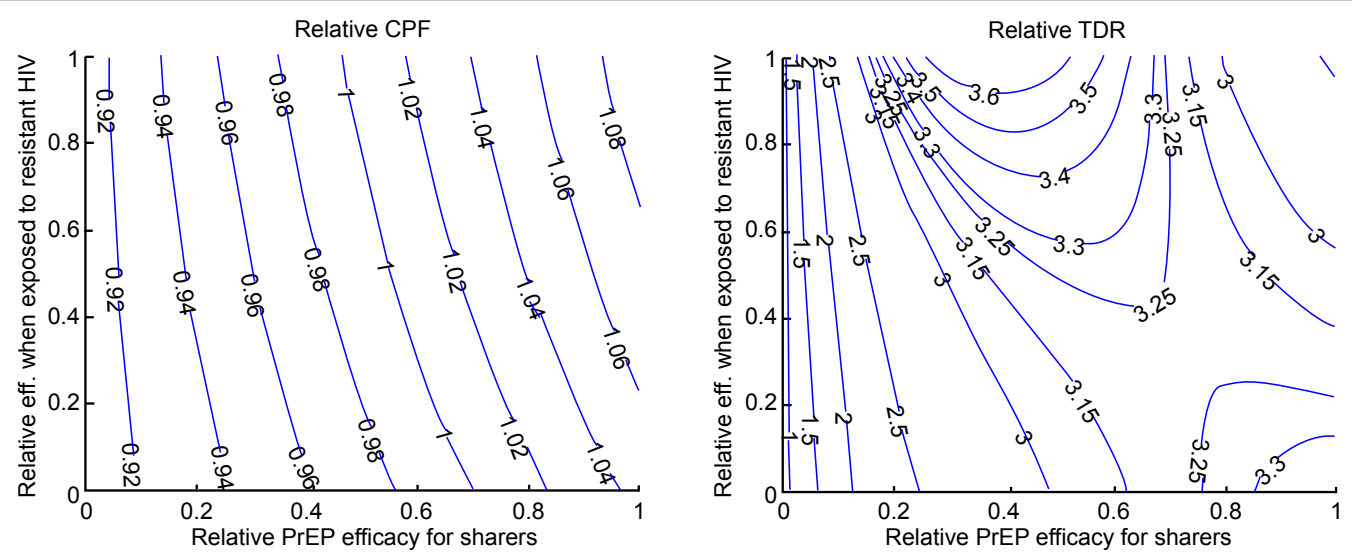

Figure 4: Impact of the relative protection ( 0 - no protection, 1 - equal protection) for sharers compared to perfect adherers and for users exposed to drug-resistant compared to wild-type HIV on A) relative CPF and B) relative TDR. Relative metrics (CPF and TDR) are calculated as the ratios of their values for scenarios with pill sharing over baseline scenario (no sharing). All other epidemic and intervention parameters are fixed on their baseline values from Table 1.
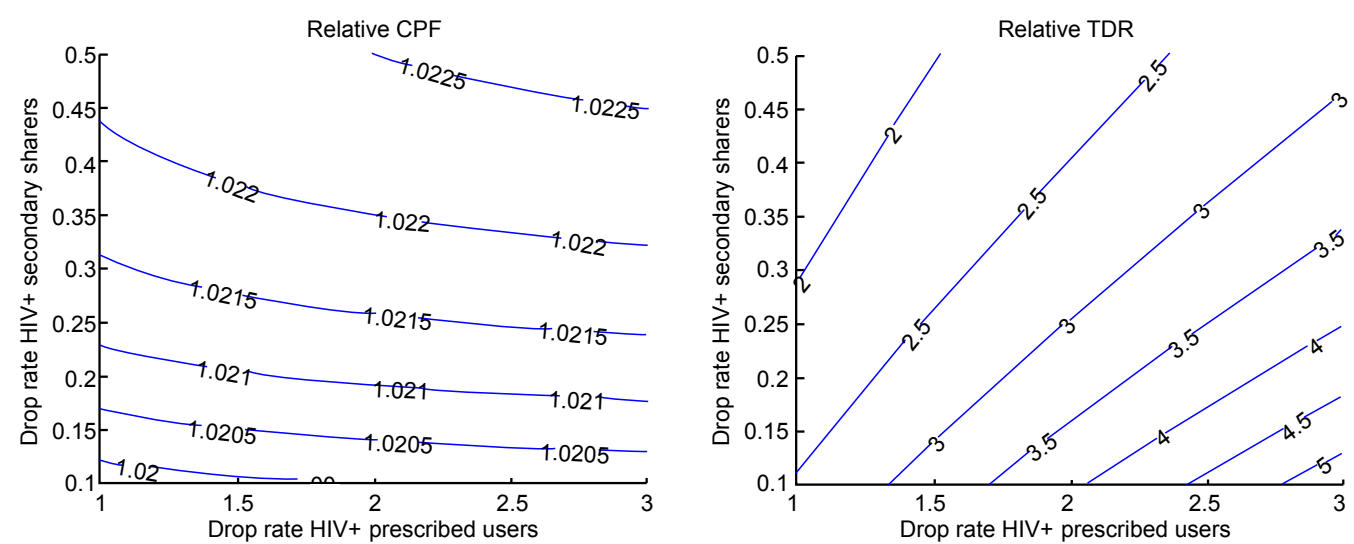

Figure 5: Impact of the annual rates at which prescribed users (perfect adherers and primary sharers) and untracked users (secondary sharers) discontinue usage of PrEP after acquiring HIV on A) relative CPF and B) relative TDR. Relative metrics (CPF and TDR) are calculated as the ratios of their values for scenarios with pill sharing over baseline scenario (no sharing). All other epidemic and intervention parameters are fixed on their baseline values from Table 1.

assumed completely inefficient for sharers ( $0 \%$ relative efficacy) due to the assumption that resistance risk is proportional to the PrEP efficacy, i.e., with no efficacy there is no selective pressure on the viral population to promote resistant HIV. Note that the most optimistic scenario with respect to $\mathrm{CPF}$, assuming $20 \%$ pill sharing and no reduction in PrEP efficacy for sharers (i.e. $100 \%$ relative efficacy), produces the largest 10year TDR of $12.3 \%$ (95\% CI 8\%-17.3\%). The total prevalence (acquired and transmitted) of drug resistance after 10 years also increases from $2 \%$ to $11 \%$ with the increase of pill sharing from $0 \%$ to $20 \%$ (Figure $3 \mathrm{C}$ ). It is not affected significantly by the variation in the PrEP protection for sharers. The fraction of the individuals with TDR after 10 years of PrEP intervention is roughly a third of all people with drug resistance regardless of the assumed population level of pill sharing (Figure 3D).

Next, we study the ratio of the intervention outcomes (CPF and TDR) for interventions with $10 \%$ sharing over baseline interventions (no pill sharing) and explore how it is influenced by the magnitude of some intervention parameters. Figure 4 presents the influence of the loss of protection due to poor adherence (suboptimal regimens) or to exposure to drug-resistant HIV. The CPF ratio varies little over the explored ranges which indicates that the CPF is very modestly influenced by PrEP efficacy among sharers and even less so by the efficacy against resistant strains, at least when only $10 \%$ are sharers
(Figure 4A). Note that pill sharing has a positive effect at reducing CPF (i.e. ratio > 1) when PrEP efficacy for sharers is relatively high (above $70 \%$ of the efficacy for perfect adherers) but detrimental when PrEP efficacy for sharers is less than $60 \%$. This indicates that the tradeoff between the increase in coverage due to sharing, i.e. more individuals are exposed to PrEP, and loss of protection for those who do not comply to the prescribed regimen may cause a delicate balance in the effects of pill sharing on the infections prevented by PrEP. On the other hand, sharing most certainly will contribute to greater fraction of the infections being resistant (Figure 4B). Our analysis predicts that PrEP needs to be completely ineffective for sharers in order to keep the TDF over 10 years at levels expected without sharing. If PrEP provides at least $40 \%$ of its efficacy to sharers a 3 -fold increase in 10 -year TDR will be attributed to pill sharing. Interestingly, the highest relative TDR levels are expected under two distinct scenarios; i) no loss of efficacy for sharers and no efficacy against resistant HIV or ii) PrEP protection for sharers is halved if compared to perfect adherers (50\% relative efficacy) and PrEP is equally effective against wild type and resistant HIV.

The effort to restrict PrEP usage by HIV-infected individuals (postintroduction control) has a negligible effect on the effectiveness of the intervention but may impact the observed incidence of transmitted drug resistance (Figure 5). Counter intuitively, the success in the 


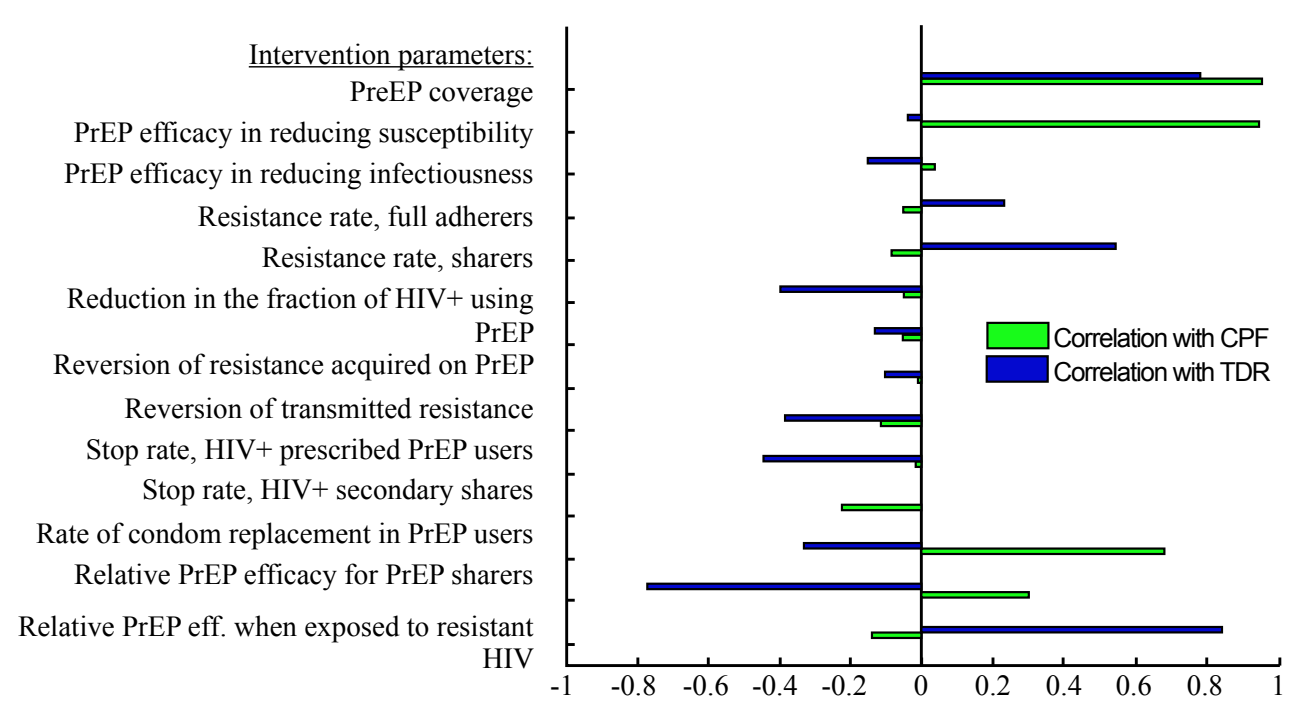

Figure 6: Partial rank correlation coefficients (PRCC) between intervention parameters and outcomes (CPF and TDR). Epidemic parameters are fixed on their baseline values from Table 1, part A.

control of prescribed users when $10 \%$ of them are primary sharers leads to larger relative TDR compared to interventions with no sharing. This may be explained by the fact that prescribed users who acquire HIV on PrEP are the only source of drug resistant cases if all users follow the prescribed regimen (no pill sharing) but its role diminishes with the increase of the proportion of users who share pills. As a result, a shortening of the period of continuous PrEP use after HIV acquisition for prescribed users have substantially stronger effect on the TDR in the scenario with no sharing and consequently contribute to the increase in the relative TDR. The improvement in the control of PrEP use by HIV-infected secondary sharers leads to decrease in relative TDR because it does not interfere with the resistance in the baseline scenario but reduces the TDR rate in the pill sharing scenarios. However, it is difficult to be achieved as part of the PrEP intervention as secondary users remain hidden from the health providers.

The influence of other modeling assumptions on the intervention outcomes (CPF and TDR) over 10 years is studied in a multivariate sensitivity analysis and the correlations between input and output parameters are presented in figure 6 . It shows that higher PrEP coverage leads to more infections prevented but also to higher levels of resistance. The proportion of users who share shows the strongest positive correlation with drug resistance while being negatively correlated with PrEP effectiveness. Biological efficacy of PrEP in reducing susceptibility is important for the CPF but has little influence on the TDR since the rate of resistance development is assumed independent of the level of PrEP protection. PrEP efficacy in reducing infectiousness has almost no impact on the intervention outcomes because of the relatively short period assumed for the prescribed users to remain on PrEP after acquiring HIV. Sensitivity analysis confirms that the relative level of PrEP protection due to sharing and due to exposure to drug-resistant HIV have a strong influence on the results and therefore need to be carefully studied before a particular PrEP intervention is rolled out. The efforts to restrict PrEP use by HIV-infected individuals through periodic testing strongly correlate with reduction in TDR but have a little influence on the CPF.

\section{Discussion}

The impact of sharing prescribed drugs on the effectiveness of HIV implementation programs is unexplored. In this study we have investigated the issue for oral ART-based PrEP interventions. Our analysis shows that pill sharing will increase the PrEP coverage level by offering an access to more users and, therefore, may have a positive impact on the observed PrEP effectiveness. On the other hand, the PrEP efficacy for users who follow suboptimal regimens may be reduced and that may compromise the impact of the intervention. In addition, pill sharing will create a pool of untracked users who do not received counseling, remain hidden and unreached by the effort to avoid PrEP usage by HIV-infected individuals. This will increase the risk for the development and spread of drug resistance that could elevate the financial burden on the public health system.

The cumulative effect of pill sharing on the PrEP effectiveness in terms of prevented infections over up to 20 year depends on the interplay between the increased PrEP coverage and the reduced protection PrEP provides for sharers. The overall effect can be positive if the efficacy reduction is relatively small since the additional protection for "new users" overcomes the loss of protection for those who share PrEP. However, if the efficacy reduction due to poor adherence is substantial ( $50 \%$ or more) the effectiveness of the PrEP intervention would likely be reduced as well.

The presence of pill sharing clearly worsens the situation with drug resistance if PrEP is used for extended periods. The prevalence of resistance and the fraction infections in which resistant HIV is transmitted (TDR) and expected to triple if $10 \%$ of prescribed PrEP users are assumed to share their drugs with a friend, a partner or a family member. The risk of resistance spread increases under all scenarios when pill sharing is practiced and may compromise the effort to control PrEP use by HIV-infected individuals. For instance, if a withdrawal from PrEP 6 months after HIV acquisition is achieved the expected resistance prevalence and the fraction of infections in which resistant HIV is transmitted remain around $2 \%$ over up to 20 years. This is consistent with the results from another modeling study [20] in which authors concluded that, whether or not risk behavior 
increased, neither the rate of emergence of resistance while on PrEP nor the testing frequency of individuals taking PrEP would have effect on increasing the number of new infections due to resistant strains. However, we showed that $10 \%$ pill sharing may be responsible for 2 to 4 -fold increase in TDR (Figure 5B). It should be pointed out that in our analysis the resistance levels are solely due to PrEP use, i.e., they should be considered in excess of the resistance due to ART failures or exposures to other preventive and treatment interventions. This analysis suggests that the effort to restrict PrEP use by HIV-infected individuals needs to be combined with a campaign targeting pill sharing practices.

The results of our analysis are limited to the specific settings included in the model. One key assumption is the mechanism of one-to-one pill sharing, i.e., one prescribed user shares with one other individual, However, we believe that this is realistic so to handle the most likely practices of the distribution of the pills between sharers: i) following lighter PrEP regimens, i.e., decreasing adherence or ii) physically splitting single pills in pieces, i.e. decreasing the dose, but keeping the prescribed regimens. The absolute impact of PrEP interventions presented in this paper is likely overestimated by the assumption that users who do not share their pills are perfect adherers. Concluded clinical trials which evaluate effectiveness of oral PrEP outline the adherence as a key factor for intervention success [37-39]. We focused on the relative impact of the pill sharing which is not likely to be affected by the overall adherence in the population if it is preserved at the same level in all intervention scenarios. Finally, our predictions are affected by the mechanisms of resistance development and their dependence on the PrEP efficacy assumed in the model. We assume that if sub-optimal usage makes PrEP less effective in preventing HIV transmission then it will also affect PrEP ability to suppress the HIV viremia which determines the selection pressure applied on the virus. Based on that we presume that the risk of drug resistance for pill sharers is reduced .No experimental data is available to justify or reject this assumption and other mechanisms could be applicable. The mechanism used in our model may underestimate the impact of pill sharing on the expected resistance especially if the risk of resistance is actually higher for sharers than for perfect adherers.

The main conclusion of this study is that the potential impact of pill sharing should not be overlooked when wide-scale intervention programs of oral PrEP are planned. Data on pill sharing and factors associated with it should be collected and monitored during the implementation stage of these programs so to better assess their impact. In addition, counseling and education materials should be provided to all users to emphasize the importance of following the prescribed regimens for maximizing the personal benefits from PrEP use.

\section{Acknowledgement}

DD and ERB are supported by a grant from the National Institutes of Health (Grant number 5 U01 Al068615-03).

\section{References}

1. World Health Organization (WHO) (2010) Unaids Report On The Global Aids Epidemic. Geneva: UNAIDS/World Health Organization.

2. Baral S, Trapence G, Motimedi F, Umar E, lipinge S, et al. (2009) HIV prevalence, risks for HIV infection, and human rights among men who have sex with men (MSM) in Malawi, Namibia, and Botswana. PloS One 4: e4997.

3. Van Griensven F, de Lind van Wijngaarden JW, Baral S, Grulich A (2009) The global epidemic of HIV infection among men who have sex with men. Curr Opin HIV AIDS 4: 300-307.

4. Abdool Karim Q, Abdool Karim SS, Frohlich JA, Grobler AC, Baxter C, et al. (2010) Effectiveness and Safety of Tenofovir Gel, an antiretroviral microbicide, for the prevention of HIV infection in women. Science 329: 1168-1174.
5. Grant RM, Lama JR, Anderson PL, McMahan V, Liu AY, et al. (2010) Preexposure Chemoprophylaxis for HIV Prevention in Men Who Have Sex with Men. N Engl J Med 363: 2587-2599.

6. Partners PrEP Study Press Release.

7. Centers of Disease Control and Prevention (2011) CDC Trial and Another Major Study Find PrEP Can Reduce Risk of HIV Infection among Heterosexuals. CDC Press Release.

8. FHI (2011) FHI Statement on the FEM-PrEP HIV Prevention Study. FHI360 Press Release.

9. Microbicide Trials Network Statement on Decision to Discontinue Use of Tenofovir Gel in VOICE, a Major HIV Prevention Study in Women.

10. Food and Drug Administration (2012) Antiviral Drugs Advisory Committee Meeting Briefing Document. Truvada, (Emtricitabine/Tenofovir Disoproxil Fumarate) For Pre-Exposure Prophylaxis of HIV-1.

11. The Consensus Committee (2012) Southern African HIV Clinicians Society, chaired by Linda-Gail Bekker and Kevin Rebe, Southern African guidelines for the safe use of pre-exposure prophylaxis in men who have sex with men who are at risk for HIV infection. South Afr J HIV Med 13: 40-55

12. Barditch-Crovo P, Deeks SG, Collier A, Safrin S, Coakley DF, et al. (2001) Phase I/II trial of the pharmacokinetics, safety, and antiretroviral activity of tenofovir disoproxil fumarate in human immunodeficiency virus-infected adults. Antimicrob Agents Chemother 45: 2733-2739.

13. Louie M, Hogan C, Hurley A, Simon V, Chung C, et al. (2003) Determining the antiviral activity of tenofovir disoproxil fumarate in treatment-naive chronically HIV-1-infected individuals. AIDS 17: 1151-1156

14. Hirsch MS, Günthard HF, Schapiro JM, Brun-Vézinet F, Clotet B, et al (2008) Antiretroviral drug resistance testing in adult HIV-1 infection: 2008 recommendations of an International AIDS Society-USA panel. Clin Infect Dis 47: $266-285$

15. Kiarie JN, Kreiss JK, Richardson BA, John-Stewart GC (2003) Compliance with antiretroviral regimens to prevent perinatal HIV-1 transmission in Kenya. AIDS 17: $65-71$

16. Weidle PJ, Wamai N, Solberg P, Liechty C, Sendagala S, et al. (2006) Adherence to antiretroviral therapy in a home-based AIDS care programme in rural Uganda. Lancet 368: 1587-1594

17. Mansergh G, Koblin BA, Colfax GN, McKirnan DJ, Flores SA, et al. (2010) Preefficacy Use and Sharing of Antiretroviral Medications to Prevent SexuallyTransmitted HIV Infection Among US Men Who Have Sex With Men. J Acquir Immune Defic Syndr 55: e14-e16.

18. Eisingerich AB, Wheelock A, Gomez GB, Garnett GP, Dybul MR, et al. (2012) Attitudes and Acceptance of Oral and Parenteral HIV Preexposure Prophylaxis among Potential User Groups: A Multinational Study. Plos One 7: e28238

19. Abbas UL, Hood G, Wetzel AW, Mellors JW (2011) Factors Influencing the Emergence and Spread of HIV Drug Resistance Arising from Rollout of Antiretroviral Pre-Exposure Prophylaxis (PrEP). PLoS ONE 6: e18165

20. Supervie V, García-Lerma JG, Heneine W, Blower S (2010) HIV, transmitted drug resistance, and the paradox of preexposure prophylaxis. Proc Natl Acad Sci U S A 107: 12381-12386

21. Pretorius C, Stover J, Bollinger L, Bacaër N, Williams B (2010) Evaluating the Cost-Effectiveness of Pre-Exposure Prophylaxis (PrEP) and Its Impact on HIV1 Transmission in South Africa. PLoS ONE 5: e13646.

22. Desai K, Sansom SL, Ackers ML, Stewart SR, Hall HI, et al. (2008) Modeling the impact of HIV chemoprophylaxis strategies among men who have sex with men in the United States: HIV infections prevented and cost-effectiveness. AIDS 22: 1829-1839.

23. Vissers DC, Voeten HA, Nagelkerke NJ, Habbema JD, de Vlas SJ (2008) The Impact of Pre-Exposure Prophylaxis (PrEP) on HIV Epidemics in Africa and India: A simulation Study. PLoS ONE 3: e2077

24. Karmon E, Potts M, Getz WM (2003) Microbicides and HIV: Help or hindrance? J Acquir Immune Defic Syndr 34: 71-75

25. Breban R, McGowan I, Topaz C, Schwartz EJ, Anton P, et al. (2006) Modeling the potential impact of rectal microbicides to reduce HIV transmission in bathhouses. Math Biosci Eng 3: 459-466

26. Vickerman P, Watts C, Delany S, Alary M, Rees H, et al. (2006) The importance 
of context: Model projections on how microbicide impact could be affected by the underlying epidemiologic and behavioral situation in 2 African settings. Sex Transm Dis 33: 397-405

27. Vickerman P, Foss A, Watts C (2008) Using Modeling to Explore the Degree to Which a Microbicide's Sexually Transmitted Infection Efficacy May Contribute to the HIV Effectiveness Measured in Phase 3 Microbicide Trials. JAIDS 48: 460-467

28. Wilson DP, Coplan PM, Wainberg MA, Blower SM (2008) The paradoxical effects of using antiretroviral-based microbicides to control HIV epidemics. Proc Natl Acad Sci U S A 105: 9835-9840

29. Dimitrov DT, Masse B, Boily M (2010) Who Will Benefit from a Wide-Scale Introduction of Vaginal Microbicides in Developing Countries? Stat Commun Infect Dis 2.

30. Boily MC, Dimitrov D, Abdool Karim SS, Mâsse B (2011) The future role of rectal and vaginal microbicides to prevent HIV infection in heterosexual populations: implications for product development and prevention. Sex Transm Infect 87 646-653

31. Kalichman SC, Simbayi LC, Cain D, Jooste S (2009) Heterosexual anal intercourse among community and clinical settings in Cape Town, South Africa. Sex Transm Infect 85: 411-415

32. Johnson L, Dorrington R, Bradshaw D, Pillay-Van Wyk V, Rehle T (2009) Sexual behaviour patterns in South Africa and their association with the spread of HIV: insights from a mathematical model. Demogr Res 21: 289-340

33. Boily MC, Baggaley RF, Mâsse B (2009) The role of heterosexual anal intercourse for HIV transmission in developing countries: are we ready to draw conclusions? Sex Transm Infect 85: 408-410

34. UNAIDS/WHO (2009) AIDS epidemic update: November 2009 Geneva: UNAIDS/World Health Organization.

35. García-Lerma JG, Cong ME, Mitchell J, Youngpairoj AS, Zheng Q, et al. (2010) Intermittent Prophylaxis with Oral Truvada Protects Macaques from Rectal SHIV Infection. Sci Transl Med 2: 14ra4

36. Prada N, Davis B, Jean-Pierre P, La Roche M, Duh FM, et al. (2008) Drugsusceptible HIV-1 infection despite intermittent fixed-dose combination tenofovir/emtricitabine as prophylaxis is associated with low-level viremia, delayed seroconversion, and an attenuated clinical course. J Acquir Immune Defic Syndr 49: 117-122

37. Donnell D, Baeten J, Hendrix C, Bumpus N, Bangsberg D, et al. (2012) Tenofovir Disoproxil Fumarate Drug Levels Indicate PrEP Use Is Strongly Correlated with HIV-1 Protective Effects: Kenya and Uganda. Abstract \#30, CROI, Seattle, WA.

38. Anderson P, Liu A, Buchbinder S, Lama J, Guanira J, et al. (2012) Intracellular Tenofovir-DP Concentrations Associated with PrEP Efficacy in MSM from iPrEx. Abstract \#31LB, CROI, Seattle, WA.

39. Van Damme L, Corneli A, Ahmed K, Agot K, Lombaard J, et al. (2012) The FEM-PrEP Trial of Emtricitabine/Tenofovir Disoproxil Fumarate (Truvada) among African Women. Abstract \#32LB, CROI, Seattle, WA

40. van de Vijver DA, Boucher CA (2010) The risk of HIV drug resistance following implementation of pre-exposure prophylaxis. Curr Opin Infect Dis 23: 621-627

41. Volberding PA, Deeks SG (2010) Antiretroviral therapy and management of HIV infection. Lancet 376: 49-62

42. Weber J, Chakraborty B, Weberova J, Miller MD, Quiñones-Mateu ME ( 2005) Diminished replicative fitness of primary human immunodeficiency virus type 1 isolates harboring the K65R mutation. J Clin Microbiol 43: 1395-1400

43. Little SJ, Frost SD, Wong JK, Smith DM, Pond SL, et al. (2008) Persistence of transmitted drug resistance among subjects with primary human immunodeficiency virus infection. J Virol 82: 5510-5518

44. Ferry B, Caraël M, Buvé A, Auvert B, Laourou M, et al. (2001) Comparison of key parameters of sexual behaviour in four African urban populations with different levels of HIV infection. AIDS 15: S41-S50

45. Morgan D, Mahe C, Mayanja B, Okongo JM, Lubega R, et al. (2002) HIV-1 infection in rural Africa: is there a difference in median time to AIDS and survival compared with that in industrialized countries? AIDS 16: 597-603

46. Porter K, Zaba B (2004) The empirical evidence for the impact of HIV on adult mortality in the developing world: data from serological studies. AIDS 18: S9S17

47. Foss AM, Hossain M, Vickerman PT, Watts CH (2007) A systematic review of published evidence on intervention impact on condom use in sub-Saharan Africa and Asia. Sex Transm Infect 83: 510-516
This article was originally published in a special issue, Drug Resistance: HIV handled by Editor(s). Dr. Claudia Goulston, University of Utah, USA 